\title{
SOME ASPECTS OF THE AGGRESSIVE ACTS, COMMITTED BY SCHIZOPHRENIC PATIENTS, TRIGGERED BY SOME PSYCHO-TRAUMATIC FACTORS
}

\author{
Stefan Todorov, Mariana Arnaoudova. \\ Department of psychiatry, Medical University - Varna; Third psychiatric Clinic, \\ University Hospital "St. Marina” - Varna, Bulgaria
}

\section{SUMMARY}

The achievements of the contemporary psychopharmacology enable the out-patient treatment of schizophrenic patients, thus let them living in their usual social environment- family, relatives, neighbors, friends.

In this connection, the real conditions of patients' micro-social environment play an important role- either stimulating their compensatory mechanisms, or exercising negative impact on their adaptation and behavior.

We examined 30 schizophrenic patients ( 22 males, 8 females), who have committed aggressive acts, triggered by psycho-traumatic situations with different sensible content, originating from their micro-social environment.

Dominant were family conflicts, followed by conflicts with the neighbors. The aggressive acts were directed to concrete persons, from the close neighborhood, involved into the psycho-traumatic situations.

Studying and evaluation of the aggressive acts, triggered by psycho-traumatic factors play an important role for their prevention

Key words: violence, aggression, schizophrenia, psycho-traumatic factors

\section{INTRODUCTION}

The achievements of the contemporary psychopharmacology enable the out-patient treatment of schizophrenic patients, thus let them living in their usual social environment- family, relatives, neighbors, friends.

A number of studies have found rates of violence to be lower in those with schizophrenia than in those with other diagnoses)- substance misuse, personality disorder and possibly other mental disorders $(1,2)$ but when compared with the general population, the evidence is overwhelmingly in favour of an increased risk of violent behaviour (3). Fazel et al. (2009) comment that some other features can play a protective role against violence in patients with schizophrenia, notably therapeutic control and compliance, family and social support, professional adaptation and better insight, whereas past violence, juvenile detention, physical abuse, parental violence, perceived threats, victimization and substance abuse are risk factors (4).

In this connection, the real conditions of patients' micro-social environment play an important role- either by stimulating their compensatory mechanisms, thus improving their psychic status and behavior and making easier their social adaptation, or exercising negative impact on their behavior, leading to worsening of their psychosis.

Violent schizophrenic patients are characterized by a particularly hostile and rejecting familial environment (5). In this situation, the risk of manifestation of different forms of social disadaptation and antisocial behavior, most often in terms of violence, increases. Serper (2011) underlines that acts of violence, committed by schizophrenic patients is a major public health concern (6). There is much evidence that schizophrenia patients have an increased risk for aggression and violent behavior due to the interrelationship between neurocognitive functioning, personality traits and violent behavior (7). As a matter of fact, a number of outstanding psychiatrists pay serious attention to the important role of the surroundings and social environment.

\section{SUBJECTS AND METHODS}

We examined 30 schizophrenic patients (22 males, 8 females), who have committed aggressive acts in terms of the relationship between psycho-traumatic situations with different sensible content, originating from their micro-social environment and the acts of violence.

\section{RESULTS AND DISCUSSION}

In 28 patients ( 20 males, 8 females) the schizophrenic psychosis proceeded the appearance of the psycho-traumatic factors, as in 7 ( 5 males, 2 females) the illness has not been diagnosed and treated. The onset of psychosis competed the aggressive acts. It could be accepted as the so called by E. Stransky (1950) "initial delict" (Das Erste delikt) (8). Humphreys et al. (1992) estimated that $20 \%$ of first-admission patients with schizophrenia had behaved in a life-threatening 
manner prior to admission (9). Volavka et al. (1997) estimated that $20 \%$ of first-contact patients with schizophrenia had assaulted another person at some time in the past (10).

The psycho-traumatic factors have had different sensible content, originating from their micro-social environment. The in-family conflicts dominated. They were provoked by intolerance from the family to the patients' behaviour, experiences, change of interests, interruption of the treatment, incapacity, etc. The sensible content of the psychic trauma has had distinct impact on patients' experiences, dynamic of psychic state and mostly on motivation for committing violent acts. Some of the more often triggers were conflicts with neighbours and friends in terms of offence, threat, attempt or battery,

With exception of 5 patients ( 2 males and 3 females), violence was mostly towards concrete persons, exerting psycho-traumatic influence on the patients, and was extremely cruel (3 homicide acts were registered).

The peculiarities of the psychopathological disorders are conditioned and different by the degree of expression of reaction towards the psycho-traumatic factor. In some cases, the psycho- traumatic factors influenced directly, whilein other cases there were latent periods with a long remodelling and incapacity to reasonable rationalization and adequate solution. We could discuss about peculiar "capsulation" of psychic trauma for a theoretically different in length periods, when the patient doesn't express any external reaction. By that time the psycho-trauma didn't loose its actuality and significant danger for violence. To a great extent, this reveals the complex genesis of the aggressive acts, committed by schizophrenic patients, triggered by psychotraumatic situation.

The causal connection was expressed in the best way in the state of remission in 18 patients (13 males, 5 females). Obviously, this could be explained by the well-known fact, that the weaker is psychopathology, the greater is the influence of different external factors, in particular, the psycho-traumatic factors and conversely- the role of outside factors is leveled when the psychotic experiences are pronounced and dominant in the clinic manifestation of schizophrenic psychosis.

\section{CONCLUSION}

The profound investigation of the violent acts, committed by schizophrenic patient, triggered by psychotraumatic factors, is of great importance for their prevention and management.

\section{REFERENCES:}

1. Harris GT, Rice ME, Quinsey VL. Violent recidivism of mentally disordered offenders: the development of a statistical prediction instrument. Criminal Justice and Behavior. 1993; 20:315 -335.

2. Wallace C, Mullen P, Burgess P, Palmer S, Ruschena D, Browne C. Serious criminal offending and mental disorder. Case linkage study. $B r J$ Psychiatry. 1998 Jun;172:477-484. [PubMed]

3. Walsch E, Buchanan A, Fachy Th. Violence and schizophrenia: examining the evidence. $B r J$ Psychiatry 2002 Jun;180:490-495. [PubMed] [CrossRef]

4. Fazel S, Gulati G, Linsell L, Geddes
JR, Grann M. Schizophrenia and violence: systematic review and metaanalysis. PLoS Med. 2009; Published on August 11. [CrossRef]

5. Ellouze F, Ayedi S, Masmoudi S, Bakri L, Chŭrif W, Zramdini R, et al. Schizophrenia and violence, incidence and risk factors: a Tunisian sample. [Article in French] Encephale. 2009 Sep;35 (4):347-52. [PubMed]

6. Serper MR. Aggression in schizophrenia. Schizophr Bull. 2011 Sep;37(5):897-898. [PubMed] [CrossRef]

7. Soyka M. Neurobiology of aggression and violence in schizophrenia. Schizophr Bull. 2011; 37(5): 913-920. [PubMed] [CrossRef]
8. Stransky E. The first offense. [Article in German] Arch Psychiatr Nervenkr Z Gesamte Neurol Psychiatr. 1950; 185(4):395-413. [PubMed]

9. Humphreys MS, Johnstone EC, MacMillan JF, Taylor PJ. Dangerous behaviour preceding first admissions for schizophrenia. Br J Psychiatry. 1992 Oct;161:501-505. [PubMed]

10. Volavka J, Laska E, Baker S, Meisner M, Czobor P, Krivelevich I. History of violent behaviour and schizophrenia in different cultures. Analyses based on the WHO study on Determinants of Outcome of Severe Mental Disorders. Br J Psychiatry 1997 Jul;171:9-14. [PubMed]

\author{
Address for correspondence: \\ Assoc. prof. Mariana Arnaoudova, MD, PhD \\ MHAT 'St. Marina”, \\ 1, Hr. Smirnenski str., Varna 9010, Bulgaria \\ phone: 00359888512627 \\ e-mail: marnaudova@hotmail.com;
}

